BULL. AUSTRAL. MATH. SOC.

VOL. 9 (1973), 169-185.

\title{
Relative annihilators \\ in semilattices
}

\section{J.C. Varlet}

An $a$-distributive (respectively a-implicative) semilattice $S$ is a lower semilattice (with greatest lower bound denoted by juxtaposition) in which the annihilator $\langle x, a\rangle$, that is $\{y \in S: x y \leq a\}$, is an ideal (respectively a principal ideal) for the fixed element $a$ and any $x$ of $S$. These semilattices appear as natural links between general and distributive semilattices on the one hand, and between pseudo-complemented and implicative semilattices on the other hand. Prime and dense elements, as well as maximal and prime filters, are essential. Mandelker's result, a lattice $L$ is distributive if and only if $(x, y)$ is an ideal for any $x, y \in L$, is extended to semilattices.

\section{Introduction}

Implicative lattices have been extensively studied by algebraists, topologists and logicians, unfortunately under various names among which we quote relatively pseudo-complemented lattices and brouwerian lattices. An implicative lattice $L$ is a set on which is defined, besides the two lattice-operations, a third binary operation, *, whose meaning is the following: for any $a, b \in L, a x \leq b \rightarrow x \leq a * b$. Since the latter involves only one of the lattice-operations, it is quite reasonable to consider implicative semilattices as in [2].

A semilattice $S$ with least element 0 is said to be pseudo-

Received 29 March 1973. 
complemented if, for any $a \in S$, there exists an element $a^{*}$ satisfying $a x=0$ if and only if $x \leq a^{*}$. Clearly any implicative semilattice bounded below is pseudo-complemented. Nevertheless, most pseudocomplemented semilattices are not implicative and furthermore an implicative semilattice need not have a zero. Whereas every implicative semilattice is distributive, this property is no longer true in a pseudocomplemented semilattice. Thus most statements are not transferable from implicative semilattices to pseudo-complemented ones and vice versa. Apart from the elementary arithmetical properties, the link between both theories is weak. In order to fill up the gap, we introduce a restricted form of implicativity, the implicativity with respect to a fixed element $a$, not necessarily zero: in an a-implicative semilattice, $x \star a$ is defined for all $x$. These semilattices have nice properties, which generalize those of pseudo-complemented semilattices and which are valid trivially in any implicative semilattice.

Extensive use is made of the notion of annihilator of an element $a$ relative to an element $b$, in symbols $\langle a, b\rangle$, introduced by Mandelker in [1]. Let us recall that the annihilator $\langle a, b\rangle$ of $a$ relative to $b$ is the family of all elements $x$ such that $a x \leq b$. Using Zorn's Lerma, we can enlarge to semilattices the nice characterization of distributive lattices given by Mandelker: a lattice $L$ (here: a semilattice) is distributive if and only if, for any $a, b \in L,\langle a, b\rangle$ is an ideal.

In Section 1 we introduce all notions and notations we need.

Section 2 is devoted to the study of the a-dense elements of a general semilattice $S$, that is, elements $x$ for which $\langle x, a\rangle$ is contained in $(a]$, the principal ideal generated by $a$. It is the natural extension of the notion of dense element in a semilattice bounded below. The properties of these elements are closely linked to those of the filters maximal with respect to the property of not containing $a$, in short: a-maximal filters.

In Section 3 we impose upon the semilattice $S$ a slight condition: $S$ has a fixed element $a$ such that $\langle x, a\rangle$ is an ideal for any $x \in S$. It is a partial distributivity that we name a-distributivity. The special case $a=0$ has already been studied by us in [5] and [6]. A characterization of $a$-distributivity in terms of $a$-maximal filters is provided and 
the subset $D(S)$ of all elements $a \in S$ for which $S$ is a-distributive is investigated. Prime elements are here of utmost importance. As already mentioned, $D(S)=S$ if and only if $S$ is distributive.

The last section deals with a-implicative semilattices, that is, semilattices $S$ in which, for any $x \in S,\langle x, a\rangle$ is a principal ideal denoted by $(x * a)$. It is proved that a semilattice $S$ is $a$-distributive if and only if its ideal lattice is (a]-implicative. The rules of computation in an $a$-implicative semilattice are given and special attention is paid to the multiplicative closure operator $\varphi$ : $x+(x * a) * a$, especially to the location of its invariant elements. Finally the congruence $\theta_{a}$ defined by $(x, y) \in \theta_{\alpha}$ if and only if $x * a=y * a$, is briefly studied.

The reader will have no difficulty in deducing properties of pseudocomplemented and implicative semilattices from our statements. In order to keep clarity and to avoid any lengthening, we do not mention these particular results: they are obvious corollaries.

\section{Preliminaries}

Throughout this paper the word semilattice will mean lower semilattice, that is a commutative idempotent semigroup or, equivalently, a partially ordered set in which any two elements $a$ and $b$ have a greatest lower bound, denoted by $a \cdot b$ or simply $a b$, the partial ordering being defined by $a \leq b \Leftrightarrow a b=a$. The greatest lower bound of the family $\left\{a_{i}: i \in I\right\}$ will be denoted by $\prod_{\{}\left\{a_{i}: i \in I\right\}$ or $\prod_{i \in I} a_{i}$ or even $\prod_{i} a_{i}$ when no confusion is allowed. The least and greatest elements of a semilattice $S$, when they exist, will be denoted by 0 and 1 respectively. When $S$ is a lattice, the second binary operation will be denoted by + .

The symbols $n, u,-, \subseteq$ and $\subset$ will be used in their usual settheoretical meaning: intersection, union, difference, inclusion and strict inclusion.

A filter of a semilattice $S$ is a non-empty subset $F$ of $S$ such that $x y \in F$ if and only if $x \in F$ and $y \in F$. The principal filter 
generated by an element $a$ of $S$, that is, the set $\{x: x \in S, x \geq a\}$, will be denoted by $[a]$. A filter $F$ of $S$ is prime if, whenever two filters $F_{1}$ and $F_{2}$ are such that $\emptyset \neq F_{1} \cap F_{2} \subseteq F$, then $F_{1}$ or $E_{2}$ belongs to $F$.

An ideal $I$ of a semilattice $S$ is a non-empty subset of $S$ satis fying

(i) $y \leq x$ and $x \in I$ imply $y \in I$;

(ii) for any $x, y \in I$, there exists $z \in I$ such that $z \geq x$ and $z \geq y$.

We shall use the symbol (a) to denote the principal ideal generated by $a$. In an up-directed semilattice $S$, a filter $F$ is prime if and only if $S-F$ is an ideal of $S$.

When ordered by inclusion, the set $F(S)$ of all filters of an updirected semilattice $S$ is a lattice in which, for any $F_{1}, E_{2} \in F(S)$, $F_{1} \cdot F_{2}=F_{1} \cap F_{2}$ and $F_{1}+F_{2}$ is the filter generated by $F_{1} \cup F_{2}$. Similar considerations are valid also for the set $T(S)$ of all ideals of $S$.

A semilattice $S$ is called modular if, for any $x, y, z$ in $S$ such that $y \geq z \geq x y$, there exists $x_{1} \geq x$ satisfying $z=x_{1} y$. The following condition is stronger: a semilattice $S$ is distributive if $z \geq x y(x, y, z \in S)$ implies the existence in $S$ of $x_{1}, y_{1}$ such that $x_{1} \geq x, y_{1} \geq y$ and $x_{1} y_{1}=z$. We proved in [6] that Stone's Separation Theorem can be extended as follows: an up-directed semilattice is distributive if and only if for any filter $F$ and any ideal $I$ such that $F \cap I=\emptyset$, there exists a prime filter containing $F$ and disjoint from $I$.

An element $a$ of a semilattice $S$ is irreducible if $a=b c$ implies $a=b$ or $a=c$. An element $a$ of $S$ is prime if (a) is a prime ideal, that is, $a \geq b c$ implies $a \geq b$ or $a \geq c$. Obviously any prime element is irreducible and the two notions coincide if $S$ is distributive.

Finally we shall make use of the notion of multiplicative closure operator. A mapping $\varphi: S \rightarrow S$ of a partially ordered set $S$ into itself 
is called a closure operator if for any $x, y \in S$,

$$
\begin{aligned}
& \text { (i) } x \leq x \varphi \text {; } \\
& \text { (ii) } x \leq y \text { implies } x \varphi \leq y \varphi \text {; } \\
& \text { (iii) }(x \varphi) \varphi=x \varphi .
\end{aligned}
$$

If the partially ordered set $S$ is endowed with a binary operation, called multiplication and denoted by juxtaposition, the closure operator $\varphi$ is termed multiplicative if, for any $x, y \in S$,

$$
\text { (iv) }(x y) \varphi=(x \varphi)(y \varphi) \text {. }
$$

\section{2. a-dense elements and a-maximal filters}

The following definition makes sense for any element $a$ of a semilattice $S$.

DEFINITION 2.1. An element $x$ of the semilattice $S$ is a-dense $(a$, fixed element of $S)$ if $\langle x, a\rangle \subseteq(a]$, that is, for every $y \in S$, $x y \leq a$ implies $y \leq a$. When $a=0$, the expression 0 -dense element is shortened to dense element.

The set of $a$-dense elements of $S$ will be denoted by $D_{a}$; when $S$ is bounded below, $D_{0}$ (shortly $D$ ) is the dense set of $S$ in the usual sense. The following facts are easily verified:

(1) $D_{a}$ is either the empty set or a filter;

(2) the following three conditions are equivalent: $a=1, D_{a} \rightarrow a$ and $D_{a}=S$;

(3) if $x$ is $a$-dense and $x y=a$, then $y=a$.

DEFINITION 2.2. A filter $F$ of the semilattice $S$ will be called a-maximal ( $a$, fixed element of $S$ distinct from 1 , if the latter exists) if $F$ is maximal with respect to the property of not containing $a$. Since $a \neq 1$, there exists $b \neq a$ and $(b) \neq a$. An easy application of Zorn's Lemma enables us to infer the existence of an a-maximal filter. Here also 0-maximal will be shortened to maximal.

THEOREM 2.3. A filter $F$ of the semilatice $S$ is a-maximal if 
and only if $F$ does not contain $a$ and, for every $x \neq F,\langle x, a\rangle$ meets $F$.

Proof $1^{\circ}$. IF: If $F$ does not contain $a$ but is not $a$-maximal, there exists a filter $G$ such that $G \supset F$ and $G \mid a$. Let us choose $x \in G-F$. By hypothesis there is $y \in F$ such that $x y \leq a$. But $x y \in G$ and $G$ would contain $a$.

$2^{\circ}$. ONLY IF: Let $F$ be an a-maximal filter. If $x \neq F$, $F+[x] \supset F$ and $F+[x] \ni a$. Hence there exists $y \in F$ such that $x y \leq a$ and $\langle x, a\rangle \cap F \neq \varnothing$.

The following statement shows that the concepts of a-dense element and a-maximal filter are closely linked.

THEOREM 2.4. In a semilatice $S$, the subset $D_{a}$ is the intersection of the a-maximal filters.

Proof. Let $\left\{F_{i}: i \in I\right\}$ be the family of a-maximal filters of $S$. We first show that, for every $i \in I, E_{i} \supseteq D_{a}$. Therefore let us suppose there is $x \in D_{a}$ but $x \neq F_{i}$ for some $i \in I$. By Theorem 2.3 one can find $y \in F_{i}$ such that $x y \leq a$. Since $x \in D_{a}$, this implies $y \leq a$ and $E_{i} \rightarrow a$, a contradiction.

Now let $b$ be an element of $\cap\left\{F_{i}: i \in I\right\}$ which does not belong to $D_{a}$. Then there exists $c$ such that $b c \leq a$ and $c \neq a$. The family of filters containing $c$ but not $a$ is not empty; by Zorn's Lemma there exists a filter $F$ maximal with respect to this property. Clearly $F=F_{i}$ for some $i \in I, b \in E, b c \in F$, which is impossible since F $\beta a \geq b c$.

THEOREM 2.5. In a semilattice $S$, the following are equivalent:

(1) $S$ has exactly one a-maximal filter;

(2) $D_{a}$ is an a-maximal filter.

Moreover they imply

(3) $D_{a}$ is a prime filter. 
Proof. By the preceding theorem the equivalence of (1) and (2) is clear since the family of a-maximal filters is totally unordered. Let us now consider a semilattice $S$ in which $D_{a}$ is an a-maximal filter. Then, for every $x \notin D_{a}$, there exists $y \in D_{a}$ satisfying $x y \leq a$. Since $y \in D_{a}$, this implies $x \leq a$. As conversely $x \leq a$ implies $x \notin D_{a}, D_{a}$ is the set-complement of the principal ideal (a] and $D_{a}$ is a prime filter.

REMARK 2.6. Generally conditions (2) and (3) are not equivalent. An example is provided by a generalized boolean lattice (that is, a distributive relatively complemented lattice bounded below but not above) to which an element 1 is added. In such a lattice, $D=(1)$ is a prime filter but is far from being a maximal filter. Nevertheless we will show in Section 4 that the previous conditions become equivalent in an a-implicative semilattice.

When the element $a$ is prime, the subset $D_{\alpha}$ takes an advantageous form.

THEOREM 2.7. In a semizattice $S$, an element $a \neq 1$ is prime if and only if $D_{a}=S-(a]$.

Proof $1^{\circ}$. IF: Let us suppose $D_{a}=S-(a)$ for some $a \neq 1$. We then have $D_{a} \neq \emptyset$. If $y z \leq a$ and $y \neq a$ (that is, $y \in D_{a}$ ), then $z \leq a$ by definition of $D_{a}$ and $a$ is prime.

$2^{\circ}$. ONLY IF: Let $a \neq 1$ be a prime element of $S$. Clearly if $x \leq a$, then $x \notin D_{a}$; on the contrary, if $x \neq a$, then $x y \leq a$ implies $y \leq a$ and $x \in D_{a}$.

\section{3. a-distributive semilattices}

DEFINITION 3.1. A semilattice $S$ is a-distributive ( $a$, fixed element of $S)$ if, for any $x \in S,\langle x, a\rangle$ is an ideal.

This definition applies to lattices as well and can be reformulated as follows: a lattice $L$ is a-distributive if, for any $x \in L, x y \leq a$ 
and $x z \leq a$ imply $x(y+z) \leq a$. Moreover $x(y+z)=a$ follows from $x y=a$ and $x z=a$. Clearly a lattice is $a$-distributive if and only if it is so as a semilattice.

The following theorem provides us with a characterization of a-distributivity in terms of filters. Its proof is similar to that of [6], Theorem 2. Nevertheless, for the sake of completeness, we give it in every detail.

THEOREM 3.2. A semilattice $S$ is a-distributive if and only if it is up-directed and any a-maximal filter is prime.

Proof $1^{\circ}$. IF: We have to prove that, for any $x \in S, I=(x, a)$ is an ideal. For any $y, z \in I$, the set of all upper bounds of $\{y, z\}$ is a filter $B$ since $S$ is up-directed. The set $G=\{t \in S: t \geq x f, f \in F\}$ is also a filter. If $G \mid a$, then $G$ is contained in an a-maximal filter $M$. By hypothesis $M$ is prime and, since $[y] \cap[z]=F \subseteq M$, either $[y] \subseteq M$ or $[z] \subseteq M$. But $y \in M$ (or $z \in M$ ) would imply (owing to $x \in M$ ) $x y \in M$ and $a \in M$, a contradiction. Hence $G \exists a$, that is, there exists $f \in F$ such that $x f \leq a$, and consequently $y, z$ have in $I$ the upper bound $f$.

$2^{\circ}$. ONLY IF: First, any a-distributive semilattice is up-directed since $(a, a)=S$ is an ideal, whence any two elements of $S$ have an upper bound. Then let $F$ be an a-maximal filter of $S$ which is not prime. There exist two filters $G$ and $H$ such that $G \cap H \subseteq F, G \notin F$ and $H \notin F$. So we can find $x \in G-F$ and $y \in H-F$. Since $F$ is $a-$ maximal, by Theorem 2.3 there exist $z$ and $t$ in $F$ belonging respectively to $(x, a)$ and $\langle y, a)$. Since $x$ and $y$ both belong to $\langle z t, a\rangle$, which is an ideal, an element $u \geq x, y$ can be found in the same annihilator, whence $z t u \leq a$. Since $z, t$ and $u$ all belong to $F, F$ contains $a$, a new contradiction.

Mandelker has shown ([1], Theorem 1 ) that a lattice $L$ is distributive if and only if it is a-distributive for all $a \in L$. Using Zorn's Lemma we can extend this property to a semilattice.

THEOREM 3.3. A semilatice $S$ is distributive if and only if it is a-distributive for all a $\in S$.

Proof $1^{\circ}$. IF: We have to prove that, for any $a, b, c$ such that 
$c \geq a b$, there exist elements $a_{1}, b_{1}$ satisfying $a_{1} \geq a, b_{1} \geq b$ and $a_{1} b_{1}=c$. Let us denote by $F_{1}$ (respectively $F_{2}$ ) the non-empty set of upper bounds of $\{a, c\}$ (respectively $\{b, c\}), F_{1}$ and $F_{2}$ are filters, as well as $F_{1}+F_{2}=\left\{z \in S: z \geq x y, x \in F_{1}, y \in F_{2}\right\}$. If $F_{1}+F_{2}$ does not contain $c$, there is a c-maximal filter $G$ containing $F_{1}+F_{2}$. Since $S$ is c-distributive, $G$ is prime by virtue of Theorem 3.2. As $\emptyset \neq F_{1}=[a) \cap[c] \subseteq G$, the element $a$ has to belong to $G$. Similarly we can show that $G$ contains $b$, whence $a b \in G$ and $c \in G$, which is impossible. In conclusion, $F_{1}+F_{2}$ does contain $c$, that is, there exist elements $d, e$ such that $d \geq a, c$ and $e \geq b, c$ together with de $\leq c$. As we also have de $\geq c$ (owing to $d \geq c$ and $e \geq c$ ), we get $c=d e$ and $d, e$ are respectively the desired elements $a_{1}, b_{1}$.

$2^{\circ}$. ONLY IF: Let $y, z \in\langle x, a\rangle$. We have to show that there is an element $t$ satisfying $t \geq y, z$ and $t \in(x, a)$. Since a distributive semilattice is up-directed, $F=[y) \cap[z]$ is a filter, as well as $G=F+[x]$. If $G \mid a$, there is a prime filter $P$ containing $G$ but not $a$. Since $P$ is prime and contains $F$, either $y \in P$ or $z \in P$. We have either $x y \in P$ or $x z \in P$, which implies $a \in P$, a contradiction. Thus we may conclude that $G \ni a$ and the existence of the desired element $t$ is established.

It is most natural to investigate the subset of elements $a$ of a semilattice $S$ for which $S$ is a-distributive. This subset, denoted by $D(S)$, enjoys the following properties.

THEOREM 3.4. In a semilatice $S, D(S)$ is a subsemilatice of $S$ including all prime elements of $S$. If $S$ is complete, then 80 is $D(s)$.

Proof. If $S$ is complete and, for every $i \in I, a_{i} \in D(S)$, then the subset $J=\left\langle x, \prod_{i} a_{i}\right\rangle$ is an ideal for every $x \in S$ and $\prod_{i} a_{i} \in D(S)$. Indeed, $y \in\left\langle x, \prod_{i} a_{i}\right\rangle$ implies $y \in\left\langle x, a_{i}\right\rangle$ for every $i \in I$. Hence if $y, a \in J$, we can find elements $t_{i}$ satisfying 
$t_{i} \geq y, z$ and $t_{i} \in\left\langle x, a_{i}\right\rangle$ for every $i \in I$. Consequently, $\prod_{i} t_{i} \in J$ with $\prod_{i} t_{i} \geq y, z$. If $s$ is not complete, the previous argument holds for I finite.

Finally, any prime element $p$ of $S$ belongs to $D(S)$ since $(x, p)$ equals $S$ or (p) according as $x \leq p$ or $x \neq p$, hence $\langle x, p\rangle$ is an ideal in both cases.

COROLLARY 3.5. Any complete semilattice in which each element has a representation as a meet of prime elements, is distributive.

Proof. The statement is a direct consequence of Theorems 3.4 and 3.3 .

REMARK 3.6. We take this opportunity to point out that, even in a complete lattice $L, D(L)$ does not include all irreducible elements of $L$. For instance, in the five-element non-modular lattice $\{0, a, b, c, 1\}$ with $0<a<b<1,0<c<1, a$ and $c$ incomparable, $b$ and $c$ incomparable, the element $a$ does not belong to $D(L)$ although it is irreducible. Actually, $\langle b, a\rangle=\{0, a, c\}$ is not an ideal.

THEOREM 3.7. In a semilattice $s$, if $a \in D(s)$, then $D_{a} \cap[a)$ is the dense set of $[a]$.

Proof. If $a=1$, the statement is trivial. Hence we may assume $a \neq 1$ in the sequel.

Let us first prove that if $x$ is a dense element of $(a)$, then $x \in D_{a}$. Let us consider an element $y$ such that $x y \leq a$. From $y \in(x, a)$ and $a \in(x, a)$ we deduce the existence of an element $z \in\langle x, a\rangle$ with $z \geq a, y$. Since $x\rangle a, z \geq a$ and $x z \leq a$, we necessarily have $x z=a$ and, owing to the density of $x$ in $[a]$, $z=a$. We conclude that $y \leq a$ and $x \in D_{a}$.

Now let us denote by $y$ an element of $D_{a} \cap[a]$ distinct from 1 . For any $z$ of $[a)$ such that $y z=a$, we have $z=a$, proving that $y$ is dense in $[a)$.

\section{4. a-implicative semilattices}

DEFINITION 4.1. A semilattice $S$ is a-implicative (a, fixed 
element of $S)$ if, for any $x \in S,(x, a)$ is a principal ideal, denoted by $(x * a]$. In other words, for any $x \in S$, we have $x y \leq a$ if and only if $y \leq x * a$.

From the point of view of universal algebra, an a-implicative algebra can be regarded as an algebra $S=\left\langle S ; \cdot,{ }^{*} a, a\right\rangle$ with a binary operation - a unary operation $x+x^{*} a=x * a$, and the distinguished element $a$.

An $a$-implicative semilattice is of course $a$-distributive and always has a reatest element 1 since $x \in\langle a, a\rangle$ is satisfied for any $x \in S$, hence $a \star a=1$; conversely, a semilattice $S$ which is bounded above is always 1 -implicative and $x * 1=1$ for any $x \in S$.

In case $S$ has a least element 0 and $S$ is 0 -implicative, it is customary to say that $S$ is preudo-complemented and to denote $x * 0$ by $x^{*}$.

A semilattice is implicative if it is a-implicative for every $a \in S$.

If $S$ is a-implicative, then $[a]$ is pseudo-complemented; if we denote by $x^{+}$the pseudo-complement of $x \quad(x \geq a)$ in $[a)$, we have $x^{+}=x * a$. The converse of this statement is not true as is easily verified.

In a semilattice satisfying the ascending chain condition, all ideals are principal. As a consequence, if such a lattice is a-distributive, it is also a-implicative. The following theorem throws light on the link between a-distributivity and a-implicativity.

THEOREM 4.2. A semilattice $S$ is a-distributive if and only if $T(S)$ is a-implicative.

Proof $1^{\circ}$. IF: We have to show that, for any $x \in S$, the subset $I=(x, a)$ is an ideal. Let $y_{1}$ and $y_{2}$ be two elements of $I$ :

$(x] \cap\left[y_{1}\right] \subseteq(a)$ and $(x] \cap\left[y_{2}\right] \subseteq(a)$. Since $T(S)$ is $(a)-$ implicative, $\left(y_{1}\right] \subseteq(x] *(a)=J$ and, similarly, $\left(y_{2}\right] \subseteq J$. As $J$ is an ideal, it contains an element $b$ such that $b \geq y_{1}, y_{2}$. From $(b] \subseteq J$ follows $(x] \cap(b] \subseteq(a]$, hence $x b \leq a$. 
$2^{\circ}$. ONLY IF: Let $I$ be an element of $T(S)$. For every $x \in I$, $J_{x}=\langle x, a\rangle$ is an ideal containing $a$, whence $J=\cap\left\{J_{x}: x \in I\right\}$ is also an ideal. We are going to prove that $I *(a]=J$. First, $I \cap J \subseteq(a]$, If not, there exist $i \in I$ and $j \in J$ such that $i j \neq a$, which is impossible given the way $J$ has been constructed. Secondly, if $k \notin J$, there is $k \in K$ such that $x k \ddagger a$ for some $x \in I$, hence $I \cap K \notin(a]$.

The following rules of computation have been proved for implicative semilattices ([2], [3], [4]). Clearly they are still valid in an a-implicative semilattice.

THEOREM 4.3. If $x, y, z$ are any elements of an a-implicative semilattice, then the following hold:

(1) $x * a \geq a$;

(2) $x \leq a, x * a=1$ and $x * a \geq x$ are equivalent;

(3) $1 * a=a$;

(4) $x * a=x$ if and only if $x=a=1$;

(5) $(x * a) * a \geq x, a$;

(6) if $x \leq y$, then $x * a \geq y * a$ and $(x * a) * a \leq(y * a) * a$;

(7) $((x * a) * a) * a=x * a$ and $(((x * a) * a) * a) * a=(x * a) * a$;

(8) $(x * a)((x * a) \star a)=a$.

As far as we know, the following important formula is novel.

THEOREM 4.4. If $x$ and $y$ are any elements of an a-implicative semilatice, then

(9) $((x y) * a) * a=((x * a) * a)((y * a) * a)$.

Proof. Let us denote by $t$ the right-side member of (9). From $x \leq(x * a) * a$ and $y \leq(y * a) * a$, we deduce $x y \leq t$, hence $(x y) * a \geq t * a$.

We now intend to prove that $(x y) * a \leq t * a$, that is, any $z$ satisfying $x y \leq a$ also satisfies $t z \leq a$. From tzxy $\leq a$ follows $\operatorname{tax} \leq y * a$. Since $\operatorname{tzx}(\leq t) \leq(y * a) * a$, we have $\operatorname{tax} \leq(y * a)((y * a) * a)=a$, hence $t z \leq x * a$. Since $t z \leq(x * a) * a$, we finally obtain $t z \leq a$ and (xy) $* a=t * a$. Then 
$((x y) * a) * a=(t * a) * a \geq t$. The opposite inclusion being trivial, the proof ends.

COROLLARY 4.5. In an a-implicative semilattice, the mopping $\varphi: x \rightarrow(2 * a) * a$ is a multiplicative closure.

Proof. Formulae (5), (6) and (7) make $\varphi$ a closure operator. The multiplicative character of this closure is given by (9).

COROLLARY 4.6. In an a-implicative algebra $s=\left(s ; \cdot,{ }^{*} a, a\right)$, the mopping $\varphi: x \rightarrow(x * a) * a$ is an idempotent endomorphism which preserves all congruences.

Proof. Theorem 4.4 shows that $(x y) \varphi=(x \varphi)(y \varphi)$. Moreover, $\left(x^{*} a\right)_{\varphi}=((x * a) * a) * a=(x \varphi)^{*} a, a \varphi=a$ and $x \varphi^{2}=x$. Finally, for any $\theta \in C(S)$, the congruence lattice of $S,(x, y) \in \theta$ implies $(x \varphi, y \varphi) \in \theta$.

DEFINITION 4.7. An element $x$ of an a-implicative semilattice $S$ will be called a-closed if $(x * a) * a=x$. The subset of a-closed elements of $S$ is denoted in the sequel by $c_{a}$. Clearly an element $x$ of an a-implicative semilattice $S$ is a-closed if and only if there is $y \in S$ satisfying $y * a=x$.

DEFINITION 4.8. A subset $A$ of a partially ordered set $S$ will be called semiconvex if $a, b \in A \quad(a \leq b)$ implies $x, y \in A$ for any $x, y$ satisfying $x y=a$ and $x+y=b$. This definition generalizes the one we introduced in [4] for lattices. Obviously a convex subset of a partially ordered set is semiconvex.

LEMMA 4.9. In a modular semilattice $S$, the subset $F_{\varphi}=\{x \in S: x \varphi=x\}$ of all $\varphi$-invariant elements of a multiplicative closure $\varphi$ is semiconvex.

Proof. Let us assume $a, b \in F_{\varphi}, 2 y=a$ and $x+y=b$. We have to show that $x \varphi=x$ and $y \varphi=y$. First $a=a \varphi=(x x)(y \varphi)$ since $\varphi$ is multiplicative. Moreover, since $x \leq x \varphi \leq b \varphi=b$ and $y \leq y \varphi \leq b \varphi=b$, clearly $2 x \varphi+y \varphi=b$. As $S$ is modular and $y \varphi \geq y \geq x(y \varphi)=a$, there is $z \in S$ satisfying $z \geq x$ and $y=z(y \varphi)$. Consequently we have $z \geq y, z \geq x+y=b \geq y \varphi$ and finally $y=y \varphi$. The inversion of the 
parts played by $x$ and $y$ gives $x=x p$. //

THEOREM 4.10. In an a-implicative algebra $s=\left\langle s ; \cdot,{ }^{*} a, a\right\rangle, c_{a}$ is a subalgebra whose least and greatest elements are $a$ and 1 respectively. If, moreover, $S$ is modular, then $C_{a}$ is semiconvex.

Proof. Since the endomorphism $\varphi: x \rightarrow(x * a) * a$ maps $S$ onto $C_{a}$, the latter is a subalgebra of $S$. Since $(a * a) \star a=a$ and $(x \star a) * a \geq a$ for any $x \in S$, the least element of $C_{a}$ is $a$. Clearly its greatest element is 1 . The last part is a direct consequence of Corollary 4.5 and Lemma 4.9 .

Our next project is to describe $a$-dense elements and a-maximal filters in an a-implicative semilattice. It is an easy exercise to verify that an element $x$ of an a-implicative semilattice is $a$-dense if and only if one of the following equivalent conditions is satisfied:

(i) $\langle x, a\rangle=(a]$;

(ii) $x * a=a$;

(iii) $(x * a) * a=1$.

THEOREM 4.11. In an a-implicative semilatice $S$, an element $x$ is a-dense if there exists $y \in S$ such that $x$ is an upper bound of $\{y, y \star a\}$.

Proof. From $x \geq y$ and $x \geq y * a$, we deduce respectively $x * a \leq y * a$ and $x * a \leq(y * a) * a$, hence $x * a \leq(y * a)((y * a) * a)=a$. Since $x * a \geq a$ elways holds, we get $x * a=a$ and $x$ is $a$-dense.

REMARK 4.12. The previous condition can be restated as follows: for every $x \in S,[x] \cap[x * a) \subseteq D_{a}$.

THEOREM 4.13. In an a-implicative semilattice $S$, a filter $F$ is a-maximal if and only if, for any $x \in S, F$ contains exactly one of the elements $x$ and $x * a$.

Proof $1^{\circ}$. IF: Since $F$ contains $1=a * a$, it does not contain $a$. Let $y \notin F$; by hypothesis $y * a \in F$. By the very definition of $y * a$ we have $y(y * a) \leq a$. Theorem 2.3 enables us to infer that $F$ is $a$-meximal. 
$2^{\circ}$. ONLY IF: Let $F$ be a-maximal. $F$ cannot contain both $x$ and $x * a$ since otherwise it would contain $a \geq x(x * a)$. Now let us suppose $x \notin F$ and $x * a \notin F$. The filter $F+[x]$ contains $a$, hence there exists $y \in F$ such that $y x \leq a$. But this means $y \leq x * a$ and $F \rightarrow x * a$, a contradiction. In conclusion, $F$ contains exactly one of the elements $x$ and $x * a$.

THEOREM 4.14. In an a-implicative semilatice $S$, a prime filter $P$ which contains $D_{a}$ but not $a$ is a-maximal.

Proof. If $x \in P$, then $x * a \mid P$ since $x(x * a) \leq a$. If $x \notin P$, $x * a \in P$ because $\emptyset \neq[x] \cap[x * a) \subseteq D_{a} \subseteq P$ jointly with $[x] \notin P$ implies $[x * a] \subseteq P$. To conclude it suffices to set forth Theorem 4.13.

COROLLARY 4.15. In an a-implicative semilattise, $D_{a}$ is a prime filter if and only if it is an a-maximal filter.

Proof. Owing to Theorem 2.5, we only need show that $D_{a}$ is $a$-maximal if it is prime. But this is straightforward by Theorem 4.14 since $D_{a} \mid a$.

Let us denote by $A(S)$ the subset of elements $a$ of $S$ for which $S$ is $a$-implicative.

THEOREM 4.16. For any semilattice $S, A(S)$ enjoys the following properties :

(1) $A(S)$ is empty if and only if $S$ has no greatest element;

(2) $\mathrm{A}(S) \subseteq D(S)$;

(3) $\mathrm{A}(S)$ is a sublattice of $S$; if $S$ is complete, so is $A(S)$;

(4) $A(S)$ includes all prime elements of $S$ if $S$ has a greatest element.

Proof. (1) and (2) are obvious. (3) is a direct consequence of the formula: $x *\left(\prod_{i \in I} a_{i}\right)=\prod_{i \in I}\left(x * a_{i}\right)$, true for any index set $I$ if $S$ is complete. In fact, if, for every $i \in I, a_{i} \in A(S)$, then we have 


$$
\begin{aligned}
x y \leq \prod_{i \in I} a_{i} & \Leftrightarrow x y \leq a_{i} \text { for every } i \in I \\
& \Leftrightarrow y \leq x \star a_{i} \text { for every } i \in I \\
& \Leftrightarrow y \leq \prod_{i \in I}\left(x \star a_{i}\right)
\end{aligned}
$$

Finally, for any prime element $p$ of $S$ we have $x * p=1$ if $x \leq p$ and $x * p=p$ if $x \neq p$.

To end with we focus our attention on a congruence of the a-implicative algebra.

THEOREM 4.17. In an a-implicative algebra $S=\left\langle s ; \cdot,{ }^{*} a, a\right\rangle$, the relation $\theta_{a}$ defined by

$$
(x, y) \in \theta_{a} \text { if and only if } x * a=y * a
$$

is a fully invariant congruence of $S$. Moreover, if $s$ is a iattice, $\theta_{a}$ is also a fully invamiant congruence of $S^{\prime}=\left(S ;+, \cdot{ }^{*} a, a\right)$.

Proof. Theorem 4.4 shows that, for every $z \in S,(x, y) \in \theta_{a}$ implies $(x z, y z) \in \theta_{a}$. Clearly $(x, y) \in \theta_{a}$ implies $(x \star a, y \star a) \in \theta_{a}$.

If $S$ is a lattice, $(x, y) \in \theta_{\alpha}$ implies $(x+z, y+z) \in \theta_{a}$ since $(x+z) \star a=(x \star a)(z \star a)$.

That $\theta_{a}$ is fully invariant is also obvious: for every $\xi \in E(S)$, the endomorphism monoid of $S,(x, y) \in \theta_{a}$ implies $x \xi * a=(x \star a) \xi=(y \star a) \xi=y \xi \star a$, hence $(x \xi, y \xi) \in \theta_{a}$.

Now we enumerate some elementary properties of $\theta_{a}$. The easy proof is left to the reader.

(1) For every $x \in S,(x,(x \star a) \star a) \in \theta_{a}$.

(2) For every $x \in S,[x] \theta_{a}$ contains exactly one element of $C_{a}$; this element is $(x * a) * a$ and constitutes the maximum element of the class. 
(3) $[a] \Theta_{a}=(a]$ is the kernel of $\Theta_{a}$ and the least element of $S / \Theta_{a} \cdot[1] \Theta_{a}=D_{a}$ is the antikernel of $\theta_{a}$ and the greatest element of $s / \Theta_{a}$.

(4) $S / \Theta_{a}$ is a boolean algebra. In fact, if we adopt the notation of 4.1, $\theta_{a} \mid[a)=\sim$, where $\sim$ is the congruence on $[a]$ defined by $(x, y) \in \sim$ if and only if $x^{+}=y^{+}$.

(5) If there exists $d \in D_{a}$ such that $x d=y d$, then $(x, y) \in \theta_{a}$.

\section{References}

[1] Mark Mandelker, "Relative annihilators in lattices", Duke Math. J. 37 (1970), 377-386.

[2] William C. Nemitz, "Implicative semi-lattices", Trans. Amer. Math. Soc. 117 (1965), 128-142.

[3] Helena Rasiowa and Roman Sikorski, The mathematics of metamathematics (Monografie Matematyczne, Tom 41. Państwowe Wydawnictwo Naukowe, Warszawa, 1963).

[4] Jules Varlet, "Contribution à l'étude des treillis pseudo-complémentés et des treillis de Stone", Mém. Soc. Roy. Sci. Liège Coll. in $8^{\circ}$ (5) 8 No. 4 (1963), 1-71.

[5] J.C. Varlet, "A generalization of the notion of pseudo-complementedness", Buzz. Soc. Roy. Sci. Liège 37 (1968), 149-158.

[6] J.C. Varlet, "Distributive semilattices and boolean lattices", Bulz. Soc. Roy. Sci. Liège 41 (1972), 5-10.

Institut de Mathématique,

Université de Liège,

8-4000, Liège,

Belgique. 